\title{
Acute mesenteric ischemia revealing cirrhosis: about a clinical case
}

\begin{abstract}
Acute mesenteric ischemia (AMI) is a rare and serious medical and surgical emergency, the prognosis of which depends on the early diagnosis and appropriate treatment. It is caused by acute or chronic interruption of splanchno-mesenteric blood flow. This interruption may be due to embolism, thrombosis or intestinal hypoperfusion. We report a case of acute mesenteric ischemia in a young subject in a National Hospital and University Center of Benin. He was a 23-year-old man with no history of admitting a diffuse abdominal pain, excruciating, sudden onset of torsion and associated vomiting and stopping of materials and gases. Emergency laparotomy revealed acute mesenteric ischemia with ileal necrosis. Ileal resection with endo-ileal endo- ileal anastomosis was performed. Etiological research has revealed cirrhosis of undetermined cause. The postoperative course was marked by short bowel syndrome and ascitic decompensation of cirrhosis. Under symptomatic treatment, vitamin and iron supplementation and diuretic evolution was favorable.
\end{abstract}

Keywords: acute mesenteric ischemia, young subject, cirrhosis
Volume II Issue 2 - 2020

\author{
Kpossou AR,' Sokpon CNM,' Doukpo MM,'² \\ Gandji EW, ${ }^{3}$ Diallo K, ${ }^{4}$ Laleye C, ${ }^{5}$ Vignon RK,' \\ Eyisse-Kpossou YOT, ${ }^{5}$ Sehonou J' \\ 'Departement of Hepato-gastroenterology, National and \\ University Hospital Hubert Koutoukou Maga (CNHU-HKM), \\ Benin \\ ${ }^{2}$ Departement of Hepato-gastroenterology, National and \\ University Hospital Hubert Koutoukou Maga (CNHU-HKM), \\ Benin \\ ${ }^{3}$ Departement of Visceral Surgery, National and University \\ Hospital Hubert Koutoukou Maga (CNHU-HKM), Benin \\ ${ }^{4}$ Department of Internal Medicine, Donka Hospital, Guinea \\ ${ }^{5}$ International Clinic of Cotonou Aupiais, Benin
} of Hepato-gastroenterology National and University Hospital Hubert Koutoukou Maga (CNHU-HKM), Cotonou, Benin, Tel 0022966I81939, Email kpossou.raimi@yahoo.fr

Received: October 24, 2020 | Published: April 03, 2020
Abbreviations: AMI, acute mesenteric ischemia; HIV, human immunodeficiency virus; $\mathrm{CT}$, computed tomography scan

\section{Introduction}

Acute mesenteric ischemia (AMI), sometimes referred to as enteric ischemia, is a diagnostic and therapeutic emergency. ${ }^{1}$ It affects the small intestine and is opposed by its topography to colonic infarction or necrotizing colitis. ${ }^{2}$ In a series of 170 patients, AMI is associated with occlusion in $34 \%$ of cases, occurs in the absence of occlusion in $60 \%$ of cases and is not documented in $6 \%$ of cases. Only rapid and multidisciplinary management can hope to improve the severity of this pathology. Mortality of AMI remains high (30 to 95\%) but depends on etiology. ${ }^{3}$ It is caused by the acute or chronic interruption of splanchno-mesenteric blood flow through mechanisms such as embolism, thrombosis or intestinal hypoperfusion. Embolic ischemia occurs from emboligenic heart disease or atheroma of the aortic arch. Ventricular aneurysms, rhythm disorders, pathology or prosthesis of mitro-aortic valves may promote thrombi formation and their peripheral embolism. Thrombotic ischemia develops in pathological digestive arteries (stenosis, atheroma, arteritis). Sometimes thrombosis occurs on a vasculitis whose main ones are the nodous periarteritis, the rheumatoid purpura, the Wegener's disease, the Churg Strauss disease, the microscopic polyangeitis, the hypereosinophilic syndrome and the Takayasu disease. Anti-phospholipid syndrome, thrombophilia or hypercoagulability may also be involved. Intestinal hypoperfusion is of multifactorial origin. It may be related to pharmacological interactions of patients suffering from multipathologies requiring many drugs which are cofactors of mesenteric vasoconstriction. The other causes of mesenteric vasoconstriction are any type of shock, dehydration, dialysis, and major cardiac or abdominal surgery, large doses of vasoconstrictor without taking into account splanchno- mesenteric hemodynamic. ${ }^{4}$ The absence of clinical signs requires the use of additional paraclinical examinations. CT scanning is currently the most effective first-line test. ${ }^{5}$ Management requires multidisciplinary collaboration and supervision in intensive care settings. The therapeutic strategy must simultaneously combine a symptomatic resuscitative component aimed at optimizing splanchnic oxygenation and a curative surgical and/or radiological component depending on the etiology of ischemia. We report a case of acute mesenteric ischemia in a young subject in a National and University Hospital of Benin; this ischemia was followed by an oedemato-ascitic decompensation revealing cirrhosis.

\section{Case presentation}

He was a 23-year-old male with no previous history, including no ethylo-smoking or cardiovascular pathology, admitted in November 2018 for diffuse, atrocious, diffuse abdominal pain, sudden twisting associated with vomiting and a cessation of materials and gases. The physical examination revealed an abdominal meteorism. The unprepared abdomen radiograph (ASP) had objectified hydro-aerobic levels wider than high. Acute intestinal obstruction by volvulus of the hail was suspected. He underwent an emergency laparotomy which revealed acute mesenteric ischemia for which an ileal resection of approximately $1.5 \mathrm{~m}$ with terminal ileo-ileal anastomosis was performed. The postoperative effects were marked by the recurrence of the $50 \mathrm{~cm}$ ileum resection and terminal ileo-ileal anastomosis. The evolution was marked by the appearance of an alteration of the general state (performance status index at 2 according to the World Health Organization); with anorexia, significant weight loss, malabsorption diarrhea made of liquid stools, at least 10/day containing food residues; with the occurrence of ascites whose puncture shows a liquid low in protein at $0.59 \mathrm{~g} / \mathrm{l}$. The patient was then transferred to 
the gastroenterology department for further assessment. On physical examination the patient had an impaired general condition with a BMI of $16.4 \mathrm{Kg} / \mathrm{m}^{2}$. The abdomen was enlarged (Figure 1) with signs of portal hypertension (collateral venous circulation, splenomegaly and ascites). Biologically, he had hepatocellular insufficiency (low prothrombin (TP) rate at $49.2 \%$, low albuminemia at $30.7 \mathrm{~g} / \mathrm{l}$ ). The electrophoresis of the proteins objectified a biological inflammation syndrome (hypoalbuminemia at $30.7 \mathrm{~g} / 1$ and hypergammaglobulinemia at $37 \mathrm{~g} / \mathrm{l})$. Esophageal endoscopy noted the presence of grade II esophageal varix without red sign with intense portal hypertension gastropathy. Abdominal CT scans (Figures 2A-C) showed atrophic liver, ascites, splenomegaly and intra-abdominal collateral venous circulation. Viral serologies B (HBsAg and total anti HBc antibodies) and $\mathrm{C}$ as well as human immunodeficiency virus (HIV) were negative. Lipid and blood sugar levels were normal. The cardiological consultation with electrocardiogram and cardiac ultrasound did not reveal any pathology. A thrombophilia check-up (antithrombin deficiency, protein $\mathrm{C}$ and $\mathrm{S}$ deficiency, Antiphospholipid antibody, factor $\mathrm{V}$ Leiden mutation, prothrombin gene mutation) was requested remotely from the ischemic episode, but not yet performed due to a lack of resources. Symptomatic treatment with analgesics (non-opioid and opioids), evacuation of ascites with diuretics, prevention of rupture of esophageal varices by beta-blocker, slowing of transit (loperamide) and nutritional assistance with oral dietary supplements and iron and calcium supplementation have been instituted. The evolution under this treatment was favourable. The patient was discharged with regular follow-up for screening for hepatocellular carcinoma.

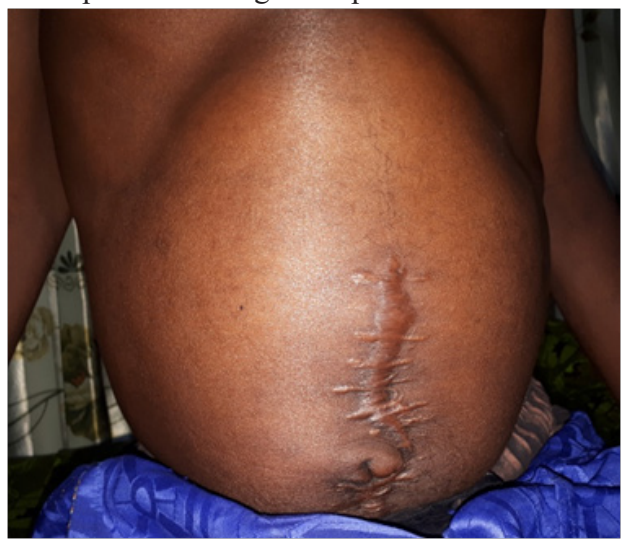

Figure I Post-operative photo of the abdomen.

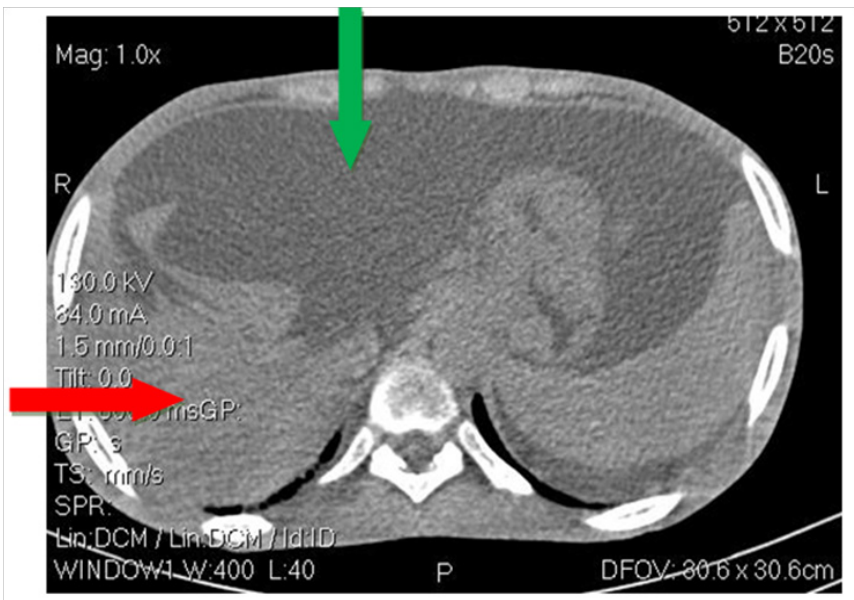

Figure 2A Abdominal CT scan showing atrophic liver and ascites.

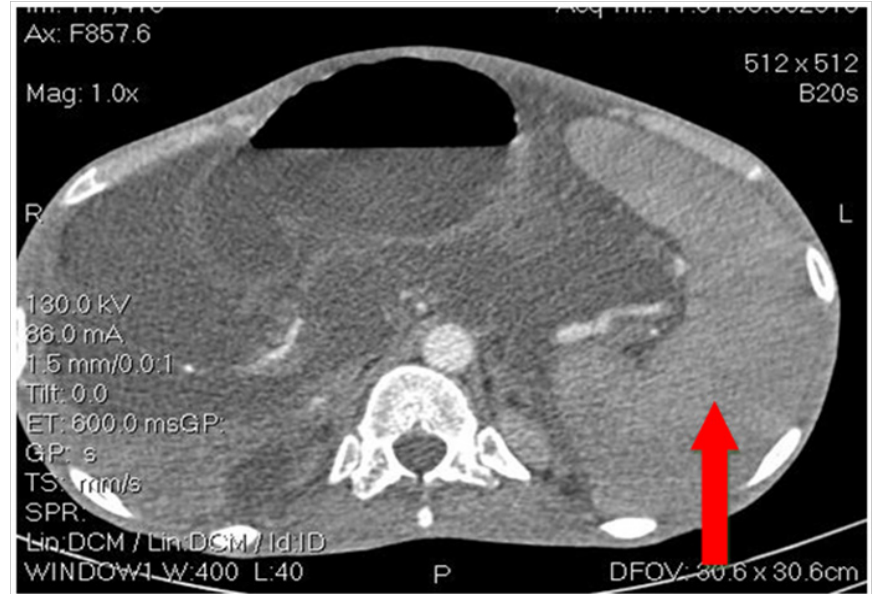

Figure 2B Abdominal CT showing splenomegaly.

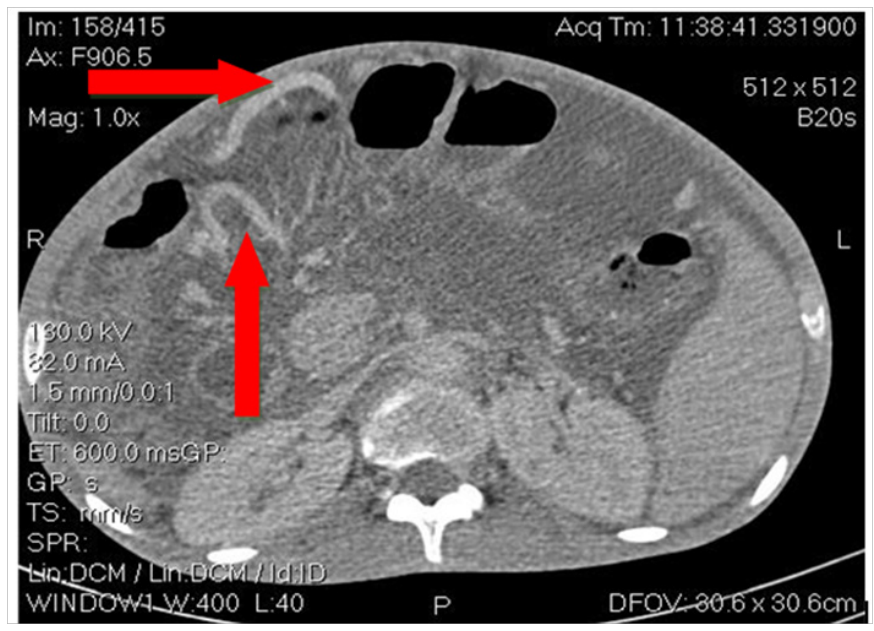

Figure 2C Abdominal CT showing collateral venous circulation.

\section{Discussion}

Intestinal ischemia (or mesenteric ischemia) is an absolute, digestive and vascular medical and surgical emergency. The evolution towards necrosis is an irreversible evolutionary shift with severe intestinal sequelae and very high mortality. ${ }^{6}$ In our clinical case, he already had intestinal necrosis, which required intestinal resection with nearly two (02) metres of small intestine removed. The clinical presentation is that of abdominal pain: it is the most sensitive sign, found in 90 to $100 \%$ of acute mesenteric ischemia. ${ }^{7}$ It is typically abrupt ("vascular") or rapidly progressive, intense and resistant to non-opioid level 2 analgesics, continuous and unrelenting, peri-umbilical or diffuse seat, and in contrast to an initially falsely reassuring abdominal palpation. Vomiting (48\%), diarrhea (31\%), digestive bleeding (18\%) may be associated with it. ${ }^{8,9}$ In some patients, the clinical presentation may mimic an acute intestinal obstruction at any point or be revealed by another ischemic disease such as alithiasis cholecystitis or splenic infarction, leading to diagnostic delays. ${ }^{10}$ In the case of our patient he was admitted with a bowel obstruction clinical presentation.

The abdominal-pelvic CT, performed without and with injection of contrast medium at arterial and portal times, is the only necessary and sufficient examination, urgently recommended for the positive diagnosis of acute mesenteric ischemia (AMI). ${ }^{6,11,12}$ It must be carried out without delay, even in the presence of renal failure, as the risk 
of ignoring an AMI far exceeds that of the injection of the contrast agent. ${ }^{6,11,13}$ It allows to characterize as well as possible the nature and morphology of vascular occlusion. ${ }^{14}$ In addition, it provides an assessment of vascular lesions and signs of intestinal necrosis that will guide revascularization treatment ${ }^{15}$ and digestive surgery ${ }^{8}$ respectively. Signs of digestive suffering include parietal thickening, parietal pneumatosis sometimes associated with an air bubble in the portal vein, infiltration of mesenteric fat, ascites. Signs of intestinal necrosis include thinning and/or failure to raise the intestinal wall and dilation of the intestinal handles $>25 \mathrm{~mm} .{ }^{8}$ The abdominal-pelvic CT could not be performed on our patient before the surgery, given the urgency of the emergency and the diagnosis was made intra operatively.

The treatment of AMI is an absolute emergency whose primary objective is to prevent or limit irreversible intestinal necrosis and its vital complications. ${ }^{8,16}$ The strategy is based on medical treatment including systematic antibiotic therapy to slow the lesional process, then systematic revascularization whenever possible, alone to heal the lesions, and finally intestinal resection according to the necrosis score, before complications occur (perforation, peritonitis). ${ }^{17}$ This is how our patient benefited in emergency from an intestinal resection due to the necrosis discovered during the operation.

The main causes of AMI are embolism, atherothrombosis, nonocclusive mesenteric ischemia, dissection, mesenteric venous thrombosis and, rarely, trauma. Embolic and thrombotic occlusions represent approximately $65 \%$ of etiologies. ${ }^{18}$ Embolism is most often of cardiac origin (atrial fibrillation, valvulopathy, left atrial dilation, recent myocardial infarction, ventricular aneurysm). ${ }^{19}$ This cause was ruled out in our patient by the cardiovascular examination and electrocardiogram, which showed no abnormalities. Similarly, atherothrombosis was ruled out in view of our patient's young age and without of obesity and dyslipidemia. Non-occlusive mesenteric ischemia occurs in patients in critical condition with low cardiac output. ${ }^{19}$ This was not the case for our patient. Mesenteric venous thrombosis accounts for about $10 \%$ of AMI. ${ }^{18}$ It is often linked to the presence of cirrhosis. ${ }^{20}$ Other etiological factors can be grouped into acquired or congenital causes. Acquired causes include neoplasia, infections, trauma and abdominal interventions, pregnancy or oral contraceptive use, Behçet's disease, myeloproliferative disease, nocturnal paroxysmal hemoglobinuria and antiphospholipid antibody syndrome. ${ }^{21}$ The congenital causes of venous ischemia are factor $\mathrm{V}$ Leiden mutation, prothrombin mutation, antithrombin deficiency and protein $\mathrm{C}$ and $\mathrm{S}$ deficiency. ${ }^{22}$

The etiology retained in our patient was cirrhosis in front of the signs of portal hypertension (collateral venous circulation, splenomegaly, esophageal varix); hepatocellular insufficiency (TP at $49.2 \%$ not corrected by vitamin $\mathrm{K} 1$ ), hepatic inflammatory syndrome (hypergammaglobulinemia) and hepatic dysmorphia (atrophic liver with bumpy contours on abdominal ultrasonography). This rare condition of cirrhosis in a young male could be explained on basis of hepatic biopsy (by transjugular route here given the coagulation disorders). But it was not feasible in our working conditions. Futhermore, the thrombophilia test has not yet been carried out; however, it should be noted that cirrhosis is often accompanied by a disruption of this test, with a protein $\mathrm{C}$ deficiency in nearly $50 \%$ of cirrhosis already at Child-Pugh stage A. ${ }^{20,22}$ The etiology of this cirrhosis is still undetermined, given the negative viral serologies $\mathrm{B}$ and $\mathrm{C}$ and the absence of any notion of alcohol consumption. Given the young age, a genetic cause (hereditary hemochromatosis, $\alpha 1$-antitrypsin deficiency) or autoimmune (autoimmune hepatitis, primary biliary cholangitis) would be possible, but limited resources did not allow further exploration. Nevertheless, the evolution has been favourable with evacuation punctures and diuretic treatment (Spironolactone) and nutritional assistance from a dietician.

\section{Conclusion}

Acute mesenteric infarction is one of the rare complications of cirrhosis. This observation also raises the problem of the difficulty of etiological research into cirrhosis in our context, once the viral and alcoholic causes have been ruled out. Liver biopsy would have been very useful for the etiological diagnosis of cirrhosis in this young subject. An improvement of our technical platform is necessary for the optimal management of patients with complex pathologies.

\section{Acknowledgments}

None.

\section{Conflicts of interest}

Author declares that there are no conflicts of interest

\section{Funding}

Funding specific to the hepatogastroenterology department of the National and University Hospital of Cotonou.

\section{References}

1. Ashraf M. Management of acute mesenteric ischemia. Arch Surg. 1999;134:328-330.

2. Goudet P, Tahan H, Sobh A, et al. Intestinal infarctions. A reevaluation of prognostic factors of postoperative mortality. Ann Chir. 1995;49(7):607-612.

3. Endean E, Barnes S, Kwolek C, et al. Surgical management of thrombotic acute intestinal ischemia. Ann Surg. 2001;233(6):801-808.

4. Corcos O, Bouhnik Y. Acute mesenteric ischemia. Post'U. 2013;177188.

5. Sievert B, Raptopoulos V, Mueller M, et al. Impact of CT on diagnosis and management of acute abdomen in patients initially treated without surgery. Am J Roentgenol. 1997;168(1):173-178.

6. Clair DG, Beach JM. Mesenteric Ischemia. $N$ Engl J Med. 2016;374(10):959-968.

7. Wyers MC. Acute mesenteric ischemia: diagnostic approach and surgicaltreatment. Semin Vasc Surg. 2010;23(1):9-20.

8. Nuzzo A, Maggiori L, Ronot M, et al. Predictive Factors of Intestinal Necrosis in Acute Mesenteric Ischemia: Prospective Study from an Intestinal Stroke Center. Am J Gastroenterol. 2017;112(4):597-605.

9. Kougias P, Lau D, El Sayed HF, et al. Determinants of mortality and treatment outcome following surgical interventions for acute mesenteric ischemia. J Vasc Surg. 2007;46(3):467-474.

10. Roth T, Mainguene C. Acute acalculous cholecystitis associated with aortic dissection: report of a case. Surg Today. 2003;33(8):633-635.

11. Oliva IB, Davarpanah AH, Rybicki FJ, et al. ACR Appropriateness Criteria (R) imaging of mesenteric ischemia. Abdom Imaging. 2013;38(4):714-719.

12. Copin P, Zins M, Nuzzo A, et al. Acute mesenteric ischemia: A critical role for the radiologist. Diagn Interv Imaging. 2018;99(3):123-134.

13. Copin P, Ronot M, Nuzzo A, et al. Inter-reader agreement of CT features of acute mesenteric ischemia. Eur J Radiol. 2018;105:87-95. 
14. Hagspiel KD, Flors L, Hanley M, et al. Computed tomography and magnetic resonance angiography imaging of the mesenteric vasculature. Tech Vasc Interv Radiol. 2015;18(1):2-13.

15. Roussel A, Castier Y, Nuzzo A, et al. Revascularization of acute mesenteric ischemia after creation of a dedicated multidisciplinary center. J Vasc Surg. 2015;62(5):1251-11256.

16. Nuzzo A, Corcos O. Management of mesenteric ischemia in the era of intestinal stroke centers: The gut and lifesaving strategy. Rev Med Interne. 2017;38(9):592-602.

17. Nuzzo A. How to optimize the management of intestinal ischemia? POST'U. 2019.

18. Björck M, Koelemay M, Acosta S, et al. Management of the diseases of mesenteric arteries and veins. Clinical Practice Guidelines of the European Society of Vascular Surgery (ESVS). Eur J Vasc Endovasc Surg. 2017;53(4):460-510.
19. Kerzmann A, Haumann A, Boesmans E, Acute mesenteric ischemia. Rev Med Liege. 2018;73(5-6):300-303.

20. Tripodi A, Mannucci PM. The coagulopathy of chronic liver disease. $N$ Engl J Med. 2011;365:147-156.

21. Parliteanu C, Gavillet M, Gié O, et al. Diagnostic et traitement des ischémies mésentériques. Rev Med Suisse. 2016;12:1419-1423.

22. Tripodi A, Anstee QM, Sogaard KK, et al. Hypercoagulability in cirrhosis: causes and consequences. J Thromb Haemost. 2011;9(9):1713-1723. 